\title{
Thermodynamics of the massive Gross-Neveu model ${ }^{*}$
}

\author{
A. Barducci, R.Casalbuoni, M. Modugno and G. Pettini \\ Dipartimento di Fisica, Univ. di Firenze \\ I.N.F.N., Sezione di Firenze \\ R. Gatto \\ Département de Physique Théorique, Univ. de Genève
}

UGVA-DPT 1994/06-854

* Partially supported by the Swiss National Foundation 


\section{ABSTRACT}

We study the thermodynamics of massive Gross-Neveu models with explicitly broken discrete or continuous chiral symmetries for finite temperature and fermion densities. The large $N$ limit is discussed bearing attention to the no-go theorems for symmetry breaking in two dimensions which apply to the massless cases. The main purpose of the study is to serve as analytical orientation for the more complex problem of chiral transition in 4-dimensional QCD with quarks. For any non-vanishing fermion mass we find, at finite densities, lines of first order phase transitions. For small mass values traces of would-be second order transitions and a tricritical point are recognizable. We study the thermodynamics of these models, and in the model with broken continuous chiral symmetry we examine the properties of the pion like state. 


\section{Introduction}

We have examined the massive Gross-Neveu model [1] at finite temperature and density within the mean field approximation. In spite of the problems related to the low dimensionality of the model, this may represent in our opinion a guide to the thermodynamics of chiral symmetry restoration in QCD. We have studied the model with a bare mass term included, always kept non vanishing. Due to such a choice, no chiral phase transition is present, since chiral symmetry is explicitly broken from the beginning and thus, strictly speaking, the Mermin-Wagner-Coleman theorem [0] [3] does not apply. Although the symmetry is explicitly broken we find, as in our previous study for a QCD model 《4, that some first order phase transition still survives. The critical line obviously moves in the plane of temperature and chemical potential for growing masses, but it survives even for large mass values. We derive the equation of state and study the phase diagram for different choices of thermodynamical variables. We discuss the isotherms in the pressure-inverse density plane, which resemble the Van der Waals isotherms for the vapour-liquid transition in the water. The construction is made through the study of the effective potential, which contains all the physical information about the stable and metastable phases of a given model. We can define a critical point as the ending point of the coexistence region. There are, below this point, two regions of very low and of very high compressibility, separated by the coexistence region which ends at the critical point.

We have also considered the explicit (small) breaking of a continuous symmetry in order to implement the study of soft-pion type properties in the model. It is evident that (differently from 4-dimensional QCD) in two dimensions the pion-like particle cannot be considered as a Goldstone particle due to the Coleman theorem. Thus one principal difference between previous studies [国] and the present work is that here the zero mass limit cannot be taken without a complete change of the picture.

To further comment on how no-go theorems work in a finite temperature field theory and to better motivate our presentation, we start by summarizing in a very schematic way known results in Section 2.

Section 3 is devoted to the results for the phase diagram and the equation of state of the model with a broken discrete symmetry. Here also naive zero mass limit results will be presented, to better clarify those for the massive case in which we are indeed interested, and at the same time to show what would be reasonable to expect in analogous studies in $3+1$ dimensions. In Section 4 we attempt at a description of pion properties in the model with a broken continuous symmetry. As the effective potential in the mean field approximation can be put in the same form as that of Section 3, the results concerning the equation of state and the phase diagram are the same. Finally, some useful calculations are summarized in the Appendix. 


\section{General review}

The Gross-Neveu model [1] is a well-known two-dimensional theory with four-fermion interactions which is asymptotically free. The fermion field has $N$ components. The model was originally considered in the $1 / N$ expansion. The massless formulations, with discrete or continuous symmetry, have been extensively studied for zero or for finite temperatures and densities [5]- [12], giving rise to several discussions related to the low dimensionality of the model, with its implications for symmetry breaking and phase transitions, and to the validity of the $1 / N$ expansion (many aspects have been already discussed in ref. [12]).

\section{Discrete symmetry}

Let us summarize in a very schematic way the main known results. The Lagrangian is

$$
\mathcal{L}=\bar{\psi} i \hat{\partial} \psi+\frac{1}{2} g^{2}(\bar{\psi} \psi)^{2}
$$

(for studies of the limit $N \rightarrow \infty$ one will also define $g^{2} N=\lambda$ ). It is invariant under the discrete chiral symmetry

$$
\psi \rightarrow \gamma_{5} \psi
$$

The four fermion interaction can be conveniently studied [1] by introducing a $\sigma$ field in the generating functional which satisfies the classical equation of motion $\sigma=g \bar{\psi} \psi$, and transforming as $\sigma \rightarrow-\sigma$ under chiral symmetry. The Lagrangian becomes

$$
\mathcal{L}=\bar{\psi} i \hat{\partial} \psi-\frac{1}{2} \sigma^{2}+g \sigma \bar{\psi} \psi
$$

and by integrating over the fermion field $\psi$ one can study the effective action as a functional of $\sigma$.

a) $T=0, \mu=0$

At zero temperature $T$ and chemical potential $\mu$ the calculation indicate the vacuum expectation value $\langle\sigma\rangle \neq 0$, and therefore the discrete chiral symmetry is spontaneously broken. This happens for any value of the coupling constant. There is no contradiction with the no-go theorems [2] [3]. 
b) $T \neq 0, \mu=0$

In this case the time dimension is bounded by $\beta=1 / T$. The thermodynamical limit can be taken only on the space dimension (unless $\beta \rightarrow \infty$ which goes back to case a)). Thus, as far as the occurrence of spontaneous symmetry breaking is concerned, the model behaves as one dimensional and the discrete chiral symmetry is immediately restored at $T_{c}=0^{+}$. This is a manifestation of the Mermin-Wagner theorem [2]. The restoration of chiral symmetry at finite temperature is driven by the presence of kinks and antikinks. These are non constant field configurations connecting the two degenerate minima, whose number grows with the volume [6]. This happens for any large, finite $N$. If however the limit $N \rightarrow \infty$ is taken before the thermodynamical limit, these configurations are suppressed, and one is left within the mean field theory, where the model exhibits a second order phase transition at a critical temperature $T_{c} \neq 0$ [5].

c) $T \neq 0, \mu \neq 0$

By considering the model in the $N \rightarrow \infty$ limit, one can derive analytically the phase diagram in the plane of chemical potential and temperature $(\mu, T)$. It turns out that there exists a tricritical point separating second order phase transitions from first order ones [9]. This result has been criticized by a lattice study [10] where the authors find that at any $\mu \neq 0$ the phase transition is first order. They claim that this is due to the formation of kink-antikink configurations which are now not suppressed and trigger the phase transition in this case.

In our study of the discrete symmetry model at finite temperature and density a bare mass is included, always taken different from zero. This ensures the elimination of kinkantikink configurations, which are suppressed in the thermodynamical limit (even in presence of a finite chemical potential t).

The model has been considered in the $N \rightarrow \infty$ limit and we believe that a $1 / N$ expansion would not destroy the qualitative picture provided the mass is always kept different from zero.

\section{Continuous symmetry}

We summarize now the main results for the model with continuous symmetry. One starts from the Lagrangian

\footnotetext{
${ }^{1}$ One can convince oneself about this fact by looking in ref. [6] at the procedure leading to the expression for the number of kinks, which is independent of the chemical potential.
} 


$$
\mathcal{L}=\bar{\psi} i \hat{\partial} \psi+\frac{1}{2} g^{2}(\bar{\psi} \psi)^{2}-\frac{1}{2} g^{2}\left(\bar{\psi} \gamma_{5} \psi\right)^{2}
$$

which is invariant under the continuous chiral transformation

$$
\psi \rightarrow e^{i \theta \gamma_{5}} \psi
$$

In this case one introduces in the generating functional, besides the scalar field $\sigma$ also a pseudoscalar field satisfying the classical equation of motion $\pi=i g \bar{\psi} \gamma_{5} \psi$ [四]. The $\sigma$ and $\pi$ fields transform under the continuous chiral transformation as

$$
\left(\begin{array}{l}
\sigma \\
\pi
\end{array}\right) \rightarrow\left(\begin{array}{cc}
\cos 2 \theta & \sin 2 \theta \\
-\sin 2 \theta & \cos 2 \theta
\end{array}\right)\left(\begin{array}{l}
\sigma \\
\pi
\end{array}\right)
$$

Integrating over the fermion fields one gets the effective action as a functional of the two fields $\sigma$ and $\pi$.

a) $T=\mu=0$

Due to chiral invariance, the effective potential is a functional of $\rho^{2} \equiv \sigma^{2}+\pi^{2}$ only. Thus one easily realizes that in the mean field approximation its analytical expression is equivalent to that of the discrete symmetry model. Then chiral symmetry appears to be spontaneously broken. This is also the result at the leading order in $1 / N$ since there is no kinetic term for the collective fields. Anyway, rigorously speaking, continuous chiral symmetry cannot be broken since there are no Goldstone bosons in two dimensions [3]. This shows up at the next-to-leading order in $1 / N$, due to IR divergencies in the $\pi$ correlation function. Nonetheless, as shown in refs. [7] [12], by choosing a polar representation for the physical fields, longitudinal and transverse

$$
\sigma+i \pi=\rho e^{i \theta}
$$

one finds that the system still allows for an "almost long range order", since the two-point correlation function decreases with a power law for large distances, the exponent being $1 / N$ [13][7]. Indeed this behaviour, which was originally found in the low-temperature phase of the continuous symmetry X-Y spin model by Kosterlitz and Thouless [13, is simply dictated by dimensional arguments. Actually it comes out from the fact that one is considering the transverse fluctuations of the order parameter, which in the IR are dominated by the free propagator of the $\pi$ field, which is $\sim 1 / k^{2}$ [1] [12]. Thus the Fourier transform in two dimensions diverges logarithmically for large distances (the same holds for the $\theta$ propagator). Anyway, by using the physical representation (2.7) this behaviour has to be exponentiated. As the hypotheses are general, the Kosterlitz-Thouless phase appears simply as the "two dimensional version of long range order" in a class of models [14]. 
A similar phenomenon occurs in $2+1$ dimensions for massless bosonic theories at finite temperature, as it appears from the behaviour of the free two point correlation function. (see Appendix D).

b) $T \neq 0$

Again, for large but finite $N$, at $T \neq 0$, one can easily find that the system behaves as in one dimension and that even this "almost long range order" disappears (see Appendix D). Anyway, even in this case, the $N \rightarrow \infty$ limit can be formally taken in a way to eliminate any space-time dependence of the correlation function. Thus one is left within the mean field description, which gives the same critical temperature as in the discrete symmetry model. This solution seems to contradict the Mermin-Wagner-Coleman theorems. We are going to comment on this point in the following.

It is evident that whenever non constant field configurations change the picture provided by the mean field approach, the $1 / N$ expansion fails. In fact the fluctuations can be suppressed only if $N$ is taken strictly infinite, rather than approximating with its leading term a $1 / N$ expansion. Thus, if $N$ is large but finite, the final result is simply that the symmetry can be broken spontaneously only if $\mathcal{D}>1$ (discrete symmetry) or $\mathcal{D}>2$ (continuous symmetry), $\mathcal{D}$ being the number of dimensions for which one takes the thermodynamical limit. It is clear that going from a zero temperature to a finite temperature field theory, $\mathcal{D}$ decreases of one unity (this will be further evidenced in the following and in Appendix D).

To specify something more about the infinite $N$, let us recall some qualitative arguments which lead to the Mermin-Wagner-Coleman results [15 for a continuous symmetry. A criterion to establish the possibility of spontaneous symmetry breaking is to consider the ratio

$$
r_{c}=\frac{Z(\alpha)}{Z(0)}=e^{-\Delta \Gamma}
$$

( $\Gamma$ is the euclidean action) between the partition function infinitesimally rotated after an operation of the symmetry group, and the unrotated one. To allow for spontaneous symmetry breaking, this ratio has to vanish in the thermodynamical limit [15], and thus $\Delta \Gamma=\Gamma(\alpha)-\Gamma(0)$ has to diverge in the thermodynamical limit. The opposite (i.e. a finite $\Delta \Gamma$ ) would imply that, being the system initially at one minimum, it would have a non zero probability to make a transition to another degenerate minimum. This would ensure the order parameter to be zero [15]. Actually, only the kinetic term contributes to $\Delta \Gamma$, which at zero temperature implies, in $D$ dimensions

$$
\Delta \Gamma \sim \int\left(\partial_{\mu} \alpha\right)^{2} d^{D} x \sim L^{D-2}
$$


which shows that if $D \leq 2$ no spontaneous symmetry breaking occurs. At finite temperature the time dimension is bounded and the difference in the actions becomes

$$
\Delta \Gamma_{\beta} \sim \int_{0}^{\beta} d x_{0} \int\left(\partial_{\mu} \alpha\right)^{2} d^{D-1} x \sim \beta L^{D-3} \sim \beta L^{d-2}
$$

where $d$ is the number of spatial dimensions. This phenomenon is much the same as the dimensional reduction at high temperatures. There, one is considering the hightemperature limit with the spatial dimensions bounded, here the large spatial-dimension limit at fixed temperature. Thus a dimensional reduction occurs whenever the ratio $x^{i} / \beta \rightarrow \infty(i=1,2, \ldots .(D-1))$.

Finally, if the theory has an internal symmetry group $O(N)$, a $N$ factorizes in the previous expressions

$$
\Delta \Gamma \sim N \beta L^{d-2}
$$

and one is lead to discuss in addition the implication of a $N \rightarrow \infty$ limit. For instance, by allowing $N \rightarrow \infty$ as $L^{\xi}$, to make $\Delta \Gamma$ divergent for $L \rightarrow \infty$ it would be enough to take $\xi>2-d$. Thus if $d=1$, the necessary condition is $\xi>1$, namely that $N$ goes to infinity faster than $L$.

The large distance behaviour of the free propagator for massless bosons in $D=d+1$

dimensions at finite temperature is dominated by $T \times($ free massless propagator in $d$ dimensions at zero temperature) (see Appendix D)

$$
D_{\beta}(\mathbf{x}) \sim T \int \frac{d^{d} k}{(2 \pi)^{d}} \frac{e^{i \mathbf{k} \cdot \mathbf{x}}}{\mathbf{k}^{2}} ; \quad \text { for }|\mathbf{x}| \rightarrow \infty
$$

Thus, as the IR leading term of the action for transverse fluctuations has the general form (2.9), the expression (2.12) leads to the same statement of eq. (2.10). In presence of an internal symmetry group $O(N)$, a factor $1 / N$ appears in (2.12), and then, by putting an IR cutoff $\sim 1 / L$, one finds that in the IR limit the fluctuations go as

$$
D_{\beta} \sim \frac{T}{N L^{d-2}} ; \quad \text { for } k \rightarrow 0
$$

which gives the same information as (2.11).

The considerations about the $N \rightarrow \infty$ limit are of course not completely satisfactory, as the limit appears a bit tricky and not physically clear. However, as the $1 / N$ expansion is non-analytic [12], to consider the $N \rightarrow \infty$ limit looks rather as moving to a new distinct model.

To summarize, several authors [5] [9] [10] have considered the massless Gross-Neveu model in view of its possible similarity to $3+1$ QCD taken in the chiral limit. Consequently they have neglected the role played by fluctuations, which can destroy the order at low dimensionality, but should not be so crucial in $3+1$. We also believe that the study of the 
model in the mean field approximation may represent a good guide to the thermodynamics of chiral symmetry restoration in 3+1 QCD models. However, both for the discrete and continuous symmetry model, we consider important to add a non vanishing bare mass term. This allows to escape the Mermin-Wagner-Coleman theorem, and besides it should represent a more realistic simulation of the QCD phase transition. We have already studied the behaviour of the condensate at finite temperature and density in a massive QCD-like theory in the mean field approach [4], with results qualitatively equivalent to those presented here. In this context it is interesting to study further properties of the Gross-Neveu model, where the technical complexity is largely reduced.

We stress again that, differently from QCD, where the zero mass limit can be safely taken, in the present case the mass has to be kept finite. In the continuous symmetry model, for instance, the illness of the zero mass limit is evident by the drastic change in the long distance behaviour of the free correlation function, which passes from an exponential decay to a logarithmic (or linear) divergence. We remark that a four fermion model in $2+1$ dimensions would a priori not be a good candidate to simulate QCD, as chiral symmetry can be properly defined only in an even number of space-time dimensions. Thus it is not surprising that the phase diagram for symmetry restoration is very different in that case [12].

In the following we will refer to the mean field results for the massless case only to better clarify the results of the massive case.

\section{The massive Gross-Neveu model - Discrete sym- metry}

In this section we examine the Gross-Neveu model when the discrete chiral symmetry is explicitly broken by adding a fermion mass term to the Lagrangian (2.1)

$$
\mathcal{L}=\bar{\psi}(i \hat{\partial}-M) \psi+\frac{1}{2} g^{2}(\bar{\psi} \psi)^{2}
$$

As already mentioned for the massless case the model is studied by introducing in the Lagrangian a $\sigma$ field satisfying the classical equation of motion $\sigma=g \bar{\psi} \psi$. In presence of a mass term it is more convenient to redefine the $\sigma$ field by shifting it by a constant $\sigma \rightarrow \sigma+M / g$. Then the Lagrangian (apart from constant terms) reads

$$
\mathcal{L}=\bar{\psi} i \hat{\partial} \psi-\frac{1}{2} \sigma^{2}+g \sigma \bar{\psi} \psi-M \frac{\sigma}{g}
$$

Let us first consider the model at vanishing temperature and density. To study the problem of spontaneous symmetry breaking (SSB) a suitable tool is the effective potential $V(\sigma)$, which has to be minimized with respect to $\sigma$ in order to extract the physically 
relevant quantities. As anticipated in the previous section, we consider the model in the infinite $N$ limit. Following ref. [16], the effective potential is obtained as

$$
V(\sigma)=\frac{1}{2} \sigma^{2}+i N \int \frac{d^{2} k}{(2 \pi)^{2}} \ln \left(g^{2} \sigma^{2}-k^{2}\right)+M \frac{\sigma}{g}
$$

The renormalization can be carried out by adding a counterterm $\delta Z \sigma^{2} / 2$ and by imposing the condition

$$
d^{2} V /\left.d \sigma^{2}\right|_{\sigma=\sigma_{0}}=1
$$

Then we have

$$
V(\sigma)=\frac{1}{2} \sigma^{2}+\frac{\lambda \sigma^{2}}{4 \pi}\left[\ln \left(\frac{\sigma^{2}}{\sigma_{0}^{2}}\right)-3\right]+M \frac{\sigma}{g}
$$

The subtraction parameter $\sigma_{0}$ is arbitrary. A change in $\sigma_{0}$ is equivalent to a change in $g$, to scaling the $\sigma$ field, and to rescaling the bare mass $M$. Thus the effective potential must obey the renormalization group equations

$$
\left(\sigma_{0} \frac{\partial}{\partial \sigma_{0}}+\beta(g) \frac{\partial}{\partial g}-\sigma \gamma_{\sigma}(g) \frac{\partial}{\partial \sigma}+M \gamma_{M}(g) \frac{\partial}{\partial M}\right) V\left(\sigma, \sigma_{0}, g, M\right)=0
$$

If the potential in eq.(3.5) is inserted into eq.(3.6) we find

$$
\begin{aligned}
\beta(g) & =-\frac{N g^{3} /(2 \pi)}{1+N g^{2} /(2 \pi)} \\
\gamma_{\sigma} & =\frac{\beta(g)}{g} \\
\gamma_{M} & =2 \gamma_{\sigma}
\end{aligned}
$$

The proportionality between $\beta(g), \gamma_{\sigma}(g)$ and $\gamma_{M}(g)$ arises from the fact that to order $1 / N$ there is no renormalization of the wave function for the $\psi$ field at the one loop level. Therefore the renormalization of $\sigma$ is determined by the term $g \sigma \bar{\psi} \psi$, that is from the renormalization of $g$.

Let us recall that for $M=0$ the symmetry is spontaneously broken and the fermion acquires a dynamical mass

$$
m_{0}=-g \hat{\sigma}=g \sigma_{0} \mathrm{e}^{1-\pi / \lambda}
$$

where $\hat{\sigma}$ is the absolute minimum of the effective potential. Eliminating the dependence of the effective potential on $\sigma_{0}$ by using the relation (3.8) between $\sigma_{0}$ and $m_{0}$, the expression (3.5) can be put in a manifestly invariant form

$$
V(\sigma)=\frac{N}{4 \pi} g^{2} \sigma^{2}\left[\ln \left(\frac{g \sigma}{m_{0}}\right)^{2}-1\right]+g \sigma \frac{M}{g^{2}}
$$


We now consider the generalization to finite temperature $T$ and chemical potential $\mu$. By using standard techniques, one can derive the effective potential

$$
\begin{aligned}
V(\sigma, T, \mu)= & V_{0}(\sigma)+V_{\beta}(\sigma, T, \mu) \\
= & \frac{N}{4 \pi} g^{2} \sigma^{2}\left[\ln \left(\frac{g \sigma}{m_{0}}\right)^{2}-1\right]+B+g \sigma \frac{M}{g^{2}} \\
& -\frac{N}{\beta} \int_{0}^{+\infty} d k\left[\ln \left(1+\mathrm{e}^{-\beta\left(\sqrt{k^{2}+g^{2} \sigma^{2}}+\mu\right)}\right)+(\mu \rightarrow-\mu)\right]
\end{aligned}
$$

As the pressure equals the negative of the effective potential evaluated at the physical points, the equation of state for the system is given by

$$
P=-V(T, \mu, \hat{\sigma}(T, \mu, M))
$$

To normalize to vanishing pressure (and energy density, in the limit $M \rightarrow 0$ ) at zero temperature and chemical potential, we have inserted in the expression for the effective potential (3.10) the "bag term" $B=N g^{2} \hat{\sigma}^{2}(0,0,0) / 4 \pi\left(\hat{\sigma}(0,0,0)=\hat{\sigma}=-m_{0} / g\right)$. Finally, it is convenient to introduce dimensionless quantities

$$
\begin{array}{lll}
\omega \equiv \frac{g \sigma}{m_{0}} & \mathcal{V} \equiv \frac{\pi}{N m_{0}^{2}} V & \alpha \equiv \frac{\pi M}{\lambda m_{0}} \\
r \equiv T / m_{0} & \eta=\mu / m_{0} & y=k / m_{0}
\end{array}
$$

and

$$
\begin{aligned}
\mathcal{V}(\omega)= & \mathcal{V}_{0}(\omega)+\mathcal{V}_{\beta}(\omega, r, \eta) \\
= & \frac{1}{4} \omega^{2}\left(\ln \omega^{2}-1\right)+\frac{1}{4}+\alpha \omega \\
& -r \int_{0}^{\infty} d y\left[\ln \left(1+\mathrm{e}^{-\frac{1}{r}\left(\sqrt{y^{2}+\omega^{2}}+\eta\right)}\right)+(\eta \rightarrow-\eta)\right]
\end{aligned}
$$

which, at the absolute minimum $\hat{\omega}$, gives the dimensionless pressure

$$
p=\frac{\pi}{N m_{0}^{2}} P=-\mathcal{V}(\hat{\omega})
$$

At zero temperature the effective potential (3.14) becomes

$$
\begin{aligned}
\mathcal{V}(\omega)_{r=0}= & \mathcal{V}_{0}(\omega)+\mathcal{V}_{\beta}(\omega, r=0, \eta) \\
= & \frac{1}{4} \omega^{2}\left(\ln \omega^{2}-1\right)+\frac{1}{4}+\alpha \omega \\
& +\frac{1}{2} \theta\left(\eta^{2}-\omega^{2}\right)\left[-\eta \sqrt{\eta^{2}-\omega^{2}}+\omega^{2} \ln \left(\sqrt{\eta^{2}-\omega^{2}}+\eta\right)-\frac{1}{2} \omega^{2} \ln \omega^{2}\right]
\end{aligned}
$$

In the following we shall consider the phase diagram and the equation of state for small values of the symmetry breaking parameter $\alpha$. 


\section{Phase diagram and thermodynamics}

At $r=\eta=0$ the symmetry is explicitly broken, and the absolute minimum of the effective potential is on the negative $\omega$-axis. It appears in correspondence of the point $\hat{\omega} \sim-(1+\alpha)$, and it becomes deeper and deeper for increasing $\alpha$, as shown in Fig. 1.

At finite $r$ and $\eta$ the symmetry still remains broken due to the presence of the breaking term $\alpha \omega$. Nevertheless, if the mass parameter is small ( $\alpha \ll 1$ but different from zero), the system at finite temperature and density exhibits a line of transitions. To be more specific, let us start by looking at the behaviour of the condensate in the plane of chemical potential and temperature $(\eta, r)$. To describe the results we find convenient to first recall the phase diagram which derives from the study of the absolute minima of the effective potential (3.14) with $\alpha=0$, already found in ref. [9] (see Fig. 2). There is a tricritical point $P_{t}=\left(\eta_{t}, r_{t}\right)=(0.608,0.318)$, separating a line of second order phase transitions $L_{I I}$ starting from the point $\left(0, r_{c}\right)=(0,0.567)$ and ending at $P_{t}$, from a first order line $L_{I}$ starting from $P_{t}$ and ending at the point $\left(\eta_{c}, 0\right)=(\sqrt{2} / 2,0)$. In the present case, with $\alpha \neq 0$, by moving along vertical lines in the $(\eta, r)$ plane, we find the following behaviour: for chemical potentials higher than a (mass dependent) value $\eta_{t}(\alpha)$ the condensate undergoes a finite discontinuity at some (mass dependent) critical temperature and drops to a value proportional to the bare mass $\alpha$. Furthermore these critical temperatures decrease for increasing chemical potentials. Thus the system still allows for "first order lines" $L_{I}(\alpha)$ starting from points $\left(\eta_{t}(\alpha), r_{t}(\alpha)\right)$ and ending at points $\left(\eta_{c}(\alpha), 0\right)$. On the other hand, for $\eta<\eta_{t}(\alpha)$, the condensate decreases continuously for growing temperatures and thus it is no longer possible to extract a line of second order critical points. Nevertheless there still exists a sharp interval of temperatures in which the condensate decreases steeply to a small value (proportional to $\alpha$ ). This simple result can be summed up by saying that by taking into account a small bare fermion mass, the phase diagram of the discrete chiral Gross-Neveu model at finite temperature and density is a perturbation of the naive mean field solution of ref. [9], found in the chiral limit. Although the last one can suffer for the presence of non constant field configurations which dominate in the thermodynamical limit, the addition of a bare mass is sufficient to eliminate these complications. The $1 / N$ corrections should not destroy the qualitative picture in this case. In Fig. 3 we show the comparison between the phase diagram for $\alpha=0$, for $\alpha=0.01$ and for $\alpha=0.1$.

In Fig. 4 we show the condensate behaviour vs. temperature at zero chemical potential for $\alpha=0,0.01,0.1$. We see that for $\alpha \ll 1$ the condensate behaviour is still reminiscent of a second order phase transition. The same holds for any $\eta<\eta_{t}(\alpha)$. The low temperature behaviour can be derived analytically and it is approximately given by

$$
\hat{\omega} \simeq-(1+\alpha)+\sqrt{2 \pi}\left(\sqrt{r}-\frac{\alpha}{\sqrt{r}}\right) \mathrm{e}^{-1 / r}
$$


In Fig. 5 we plot the condensate behaviour vs. chemical potential at zero temperature for $\alpha=0,0.01,0.1$, as derived from the effective potential given by eq.(3.16). In this case the critical chemical potential can be evaluated analytically for $\alpha \ll 1$. It turns out to be

$$
\eta_{c}(\alpha) \sim \frac{\sqrt{2}}{2}(1+2 \alpha)
$$

Let us now discuss the equation of state. From eqs.(3.15) and (3.14) we have

$$
\begin{aligned}
p(r, \eta)= & \frac{\hat{\omega}^{2}}{4}\left(1-\ln \hat{\omega}^{2}\right)-\frac{1}{4}-\alpha \hat{\omega} \\
& +r \int_{0}^{\infty} d y \ln \left[1+e^{-\left(\sqrt{y^{2}+\hat{\omega}^{2}}+\eta\right) / r}\right]+(\eta \rightarrow-\eta)
\end{aligned}
$$

(we recall that the minimum $\hat{\omega}$ depends on $r, \eta$ and $\alpha$ ).

The fermion number density and the entropy density are obtained by the derivatives of the pressure $P$ with respect to the chemical potential and temperature respectively. However it is convenient to define dimensionless number- and entropy-densities

$$
\begin{aligned}
n & =\frac{\partial p}{\partial \eta} \\
& =\int_{0}^{\infty} \frac{d y}{1+e^{\left[\sqrt{y^{2}+\hat{\omega}^{2}}-\eta\right] / r}}-(\eta \rightarrow-\eta)
\end{aligned}
$$

and

$$
\begin{aligned}
s & =\frac{\partial p}{\partial r} \\
& =\int_{0}^{\infty}\left[\left(\frac{1}{r}\right) \frac{\sqrt{y^{2}+\hat{\omega}^{2}}+\eta}{1+e^{\left[\sqrt{y^{2}+\hat{\omega}^{2}}+\eta\right] / r}}+\ln \left(1+e^{-\left[\sqrt{y^{2}+\hat{\omega}^{2}}+\eta\right] / r}\right)\right]+(\eta \rightarrow-\eta)
\end{aligned}
$$

which are the true quantities rescaled by a factor $\pi /\left(N m_{0}\right)$. Consistently, the dimensionless energy density is given by the expression

$$
\begin{aligned}
\varepsilon & =-p+r \frac{\partial p}{\partial r}+\eta \frac{\partial p}{\partial \eta} \\
& =-p+r s+\eta n \\
& =\frac{\hat{\omega}^{2}}{4}\left(\ln \hat{\omega}^{2}-1\right)+\frac{1}{4}+\alpha \hat{\omega}+\int_{0}^{\infty} \frac{\sqrt{y^{2}+\hat{\omega}^{2}}}{1+e^{\left[\sqrt{y^{2}+\hat{\omega}^{2}}+\eta\right] / r}}+(\eta \rightarrow-\eta)
\end{aligned}
$$

As we have previously done for the phase diagram, it is useful first to refer to the results deriving from (3.14) with $\alpha=0$. In this case the exact values of $p, n, s, \varepsilon$ can be derived analytically at $r=\eta=0$ and for $r>r_{c}(\eta)$ (where $r_{c}(\eta)$ is a point of the curves $L_{I I}(\alpha=0)$ or $L_{I}(\alpha=0)$ ). In fact, for $\alpha=0$ and $r=\eta=0$, from (3.14), one has $\hat{\omega}=1$, and thus from eqs.(3.19)-(3.22) $p=s=n=\varepsilon=0$, whereas for $r>r_{c}(\eta)$, one has $\hat{\omega}=0$, and thus 
the integrals in eqs.(3.19)-(3.22) can be exactly evaluated, giving

$$
\begin{aligned}
p & =\frac{\pi^{2} r^{2}}{6}+\frac{\eta^{2}}{2}-b \\
s & =\frac{\pi^{2} r}{3} \\
n & =\eta \\
\varepsilon & =p+2 b
\end{aligned}
$$

(where $b=1 / 4$ is the dimensionless bag term).

In Fig. 6 we show the curves $\varepsilon / r^{2}$ and $p / r^{2}$ versus $r$, for $\eta=0$ and $\alpha=0$. Notice that for $r>r_{c} \simeq 0.57$ the two curves approach asymptotically the constant value $K=\pi^{2} / 6$. In fact their expression in this region is given by

$$
\begin{aligned}
& \frac{p}{r^{2}}=\frac{\pi^{2}}{6}+\frac{\eta^{2}}{2 r^{2}}-\frac{b}{r^{2}} \\
& \frac{\varepsilon}{r^{2}}=\frac{\pi^{2}}{6}+\frac{\eta^{2}}{2 r^{2}}+\frac{b}{r^{2}}
\end{aligned}
$$

and thus for $\eta=0$, the two curves in this "Stefan-Boltzmann regime", are symmetrical with respect to the dashed line depicted in Fig. 6. Notice also that since both $\varepsilon$ and $p$ are exponentially vanishing for $r \rightarrow 0$, their ratio to $r^{2}$ is vanishing too.

In Fig. 7 there are the same quantities as in Fig. 6, for $\eta=0.63>\eta_{t}(\alpha=0) \simeq 0.608$. Notice that in this case the curve of the energy density shows a latent heat as the phase transition is first order, and that the two curves are no longer symmetrical with respect to the asymptotic value $K$.

For $\alpha \neq 0$, the pressure and energy density at $r=\eta=0$ are corrected to $p \simeq \alpha$ and $\varepsilon \simeq-\alpha$. This is expected because the energy density has to be proportional to the condensate, which means $\varepsilon=\alpha \hat{\omega} \simeq-\alpha$ since $\hat{\omega} \sim-(1+\alpha)$ at $r=\eta=0$. A consequence is that at low temperatures the ratios $p / r^{2}$ and $\varepsilon / r^{2}$ are now divergent. Thus, to construct curves analogous to those in Figs. 6 and 7 (where $\alpha=0$ ) for the massive case, we have to subtract the divergence. Actually in Figs. 8 and 9 we show $[\varepsilon+\alpha] / r^{2}$ and $[p-\alpha] / r^{2}$ vs. $r$ at fixed $\eta$ with $\eta=0<\eta_{t}(\alpha)$ and $\eta=0.65>\eta_{t}(\alpha)$ respectively, for $\alpha=0.01$. Notice that the latent heat is slightly reduced with respect to the massless case.

We now come to alternative representations of the phase diagram, different from those of Figs. 2 and 3. We consider the planes $(1 / n, p)$ and $(n, \varepsilon)$, of inverse fermion density-pressure and of fermion density-energy density, which are quantities of more direct physical interpretation than $r$ and $\eta$.

Let us start from the isotherms in the $(1 / n, p)$ plane for the massless model, in Fig. 10. These van der Waals type curves are readily obtained in the following way: the critical line $L_{I I}$ is the mapping of the corresponding curve in the ( $\left.\eta, r\right)$ plane (see Fig. 2), 
whereas the line $L_{I}$ has split into two parts, corresponding to the value as of the density $n$ at the two degenerate minima at the critical chemical potential at a given temperature for $r<r_{t}$. The region inside is the coexistence region. Notice that it is not necessary to follow the Maxwell procedure to draw the isotherms, as all the informations are naturally included in the formalism. In fact the value of the pressure at the boundaries of the coexistence region for a given temperature is directly the negative of the value of the effective potential at the two degenerate minima at the critical chemical potential. The "liquid phase" corresponds to the region where "the symmetry is restored" and thus where the absolute minimum is $\hat{\omega}=0$, and the "gas phase" corresponds to the region where "the symmetry is broken", or $\hat{\omega} \neq 0$. The intermediate values of $n$ are of course not accessible from the minima of the effective potential as they only give informations concerning the pure phases. They are however physically accessible, in analogy, for instance, with the phenomenon of the melting of ice at zero temperature. The supercooled and overheated parts of the isotherms can be easily obtained by following respectively the histories of the minima at the origin and out of the origin, after they cease to be absolute minima. The unstable region is obtained by following the maximum in between, since it appears together with one of the two minima until it merges with the other. The picture obtained in this way obeys the Maxwell construction which is simply derived from the equality of the chemical potentials at the edge of the two phases.

The isotherms in the symmetric phase have a simple analytical expression for $\alpha=0$. In fact, from eqs. (3.23) and (3.25) which is $n=\eta$ in the symmetric phase, one has

$$
p=\frac{\pi^{2} r^{2}}{6}+\frac{1}{2(1 / n)^{2}}-b \quad \text { (symmetric phase) }
$$

The isotherms in the "gas phase" have been obtained numerically.

The curve $\gamma$ in Fig. 10 is the isotherm at zero temperature, and therefore it defines the edge of the accessible region. From the point $C=\left(1 / n_{c}, 0\right)=(\sqrt{2}, 0)$ it coincides with the horizontal axis (up to infinity as $n=0$ for $\eta<\eta_{c}$ ). Thus the line $\gamma$ has the simple expression

$$
p=\theta\left(\sqrt{2}-\frac{1}{n}\right) \frac{1}{2}\left(\frac{1}{\left(1 / n^{2}\right)}-\frac{1}{2}\right)
$$

In Fig. 11 are shown the isotherms in the plane of inverse density and pressure for $\alpha=0.01$, as derived numerically. The change in the shape of the coexistence region, close to the critical point $P_{t}$, is well understood by classical arguments of a Landau expansion (see Appendix A) [17].

As one can see, the description for $\alpha \neq 0(\alpha \ll 1)$ is strictly related to that for the massless case. This is not surprising as the model admits a Landau expansion which does not involve problems related to long range fluctuations if $\alpha \neq 0$. Thus the system still shows up in two different phases below the critical pressure $p_{t}$. The situation for $\alpha \neq 0$ 
is much the same as for the vapour-liquid transition for water. It is possible to obtain Clausius-Clapeyron-like equations from the equality of the pressure at the edge of the two phases (see ref.[18]).

Finally we come to the phase diagram in the plane $(n, \varepsilon)$, starting from the case $\alpha=0$ in Fig. 12. The picture clearly indicates the different regions of chiral symmetry breaking and restoration, and the region of coexistence, by the mapping of the corresponding lines in Fig. 10. Here the only isotherm drawn is the line $\gamma$, which is that for zero temperature, and thus it defines the edge of the accessible region (as in Fig. 10). It has the simple expression

$$
\varepsilon=\theta\left(\frac{\sqrt{2}}{2}-n\right) \frac{\sqrt{2}}{2} n+\theta\left(n-\frac{\sqrt{2}}{2}\right)\left(\frac{1}{4}+\frac{n^{2}}{2}\right)
$$

The second term in the r.h.s. is obtained from eqs. (3.23), (3.25) and (3.26) by taking into account that $n_{c}=\eta_{c}=\sqrt{2} / 2$ and $b=1 / 4$. As far as the first term is concerned, let us first recall that, at zero temperature (see also the second line of eq.(3.22))

$$
\varepsilon=-p+\eta n
$$

As the phase transition at $r=0$ is first order, the previous formula directly gives the latent heat in passing from one phase to the other

$$
\operatorname{disc} \varepsilon=\eta_{\mathrm{c}} \operatorname{disc} \mathrm{n}
$$

(namely the coordinates of the point $C=(\sqrt{2} / 2,1 / 2)$ ). By allowing for the intermediate values of $n$ (which correspond to the physical values in the mixed phase) and taking into account that $p_{c}\left(\eta_{c}, r=0\right)=0$, whereas the "pure phase" values of $n$ are $n_{1}=0$, $n_{2}=\sqrt{2} / 2$, one gets a straight line

$$
\varepsilon=-p_{c}+\eta_{c} n=\theta\left(\frac{\sqrt{2}}{2}-n\right) \frac{\sqrt{2}}{2} n
$$

which is the first term in the r.h.s. of eq. (3.31).

In Fig. 13 there is the phase diagram in the $(n, \varepsilon)$ plane for $\alpha=0.01$. Also from this picture (as in Fig. 11), apart from the obvious disappearing of the line $L_{I I}$, the strong effect of the addition of a bare mass on the shape of the curve in the vicinity of the point $P_{t}$ becomes evident.

\section{Continuous symmetry}

In this section we consider the massive Gross-Neveu model described by the Lagrangian

$$
\mathcal{L}=\bar{\psi}(i \hat{\partial}-M) \psi+\frac{1}{2} g^{2}(\bar{\psi} \psi)^{2}-\frac{1}{2} g^{2}\left(\bar{\psi} \gamma^{5} \psi\right)^{2}
$$


which for $M=0$ is invariant under the continuous chiral transformation (2.5). With $M \neq 0$ the symmetry is broken from the beginning and there is no reason for the appearance of exact Goldstone bosons or exact massless particles in general. Actually, as far as the phase diagram and the thermodynamics is concerned, it is easy to realize that the study of the model in the present case leads to the same results shown in the previous section. In fact, after having introduced scalar and pseudoscalar fields and integrated over the fermion fields, following the same procedure of the previous section, we obtain an effective potential of the form

$$
V\left(\rho^{2}, \sigma, T, \mu\right)=V^{(0)}\left(\rho^{2}\right)+g \sigma \frac{M}{g^{2}} ; \quad\left(\rho^{2}=\sigma^{2}+\pi^{2}\right)
$$

where $V^{(0)}\left(\rho^{2}\right)$ has the same form of $(3.10)$ with $M=0$ and $\sigma \leftrightarrow \rho$ By imposing the minimum conditions

$$
\left.\frac{d V}{d \sigma}\right|_{(\sigma, \pi)=(\hat{\sigma}, \hat{\pi})}=0=\left.\frac{d V}{d \pi}\right|_{(\sigma, \pi)=(\hat{\sigma}, \hat{\pi})}
$$

it comes out that

$$
\begin{gathered}
\left.2 \sigma \frac{\partial V^{(0)}}{\partial \rho^{2}}\right|_{(\sigma, \pi)=(\hat{\sigma}, \hat{\pi})}+\frac{M}{g}=0 \\
\left.2 \pi \frac{\partial V^{(0)}}{\partial \rho^{2}}\right|_{(\sigma, \pi)=(\hat{\sigma}, \hat{\pi})}=0
\end{gathered}
$$

and thus $\hat{\pi}=0$, and the effective potential reduces to the one obtained in Section 3 .

\section{Pion decay constant}

Our aim now is to analyse the pion mass and decay constant behaviours in $T$ and $\mu$ in the small mass limit. First of all we notice that the axial current $\mathcal{J}_{\mu}^{5}=\bar{\psi} \gamma_{\mu} \gamma^{5} \psi$ formally satisfies the PCAC relation

$$
\partial^{\mu} \mathcal{J}_{\mu}^{5}=2 \frac{M}{g} \pi
$$

Moreover, for dimensional reasons, $\pi$ cannot be identical to the canonical pion field. Indeed we expect that the physical pion appears in the Lagrangian with a term $c_{0}^{2} \varphi_{\pi}^{2}$, where $c_{0}$ has the dimension of a mass. Therefore we impose that the renormalized propagator for $\varphi_{\pi}$ has the canonical form

$$
D_{\varphi_{\pi}}(x)=\left\langle T \varphi_{\pi}(x) \varphi_{\pi}(0)\right\rangle=\int \frac{d^{2} p}{(2 \pi)^{2}} \frac{i}{p^{2}-m_{\pi}^{2}} \mathrm{e}^{i p \cdot x}
$$

Comparing this expression with $D_{\pi}(x)=\langle T \pi(x) \pi(0)\rangle$, we obtain that $c_{0}$ is the residue of $D_{\pi}\left(p^{2}\right)$ at the pole corresponding to the pion mass $\mathrm{z}$

$$
c_{o}^{2}=\left.\operatorname{Res} D_{\pi}\left(p^{2}\right)\right|_{p^{2}=m_{\pi}^{2}}
$$

\footnotetext{
${ }^{2}$ And thus $g c_{o}$ is invariant under the renormalization group.
} 
The divergence of the axial current then becomes

$$
\partial^{\mu} \mathcal{J}_{\mu}^{5}=\frac{2 M c_{o}}{g} \varphi_{\pi}
$$

Defining in the usual way the pion decay constant

$$
\left\langle 0\left|\mathcal{J}_{\mu}^{5}(0)\right| \varphi_{\pi}\right\rangle \equiv i f_{\pi} p_{\mu}
$$

we have

$$
\begin{gathered}
m_{\pi}^{2} f_{\pi}=\frac{2 M c_{o}}{g} \\
\partial^{\mu} \mathcal{J}_{\mu}^{5}=m_{\pi}^{2} f_{\pi} \varphi_{\pi}
\end{gathered}
$$

In the soft pion limity (at $T, \mu=0$ ) the pion mass can be extracted in the following way 21]

$$
m_{\pi}^{2}=-i c_{o}^{2} D_{\pi}^{-1}(0)=\left.c_{o}^{2} \frac{\partial^{2} V}{\partial \pi^{2}}\right|_{\min }=\left.\frac{\partial^{2} V}{\partial \varphi_{\pi}^{2}}\right|_{\min }
$$

where $c_{o}^{2}$ is the residue of $D_{\pi}\left(p^{2}\right)$ on the pole.

At finite $T, \mu$, defining $m_{\pi}^{2}(T, \mu)$ as the pole of the thermal two-point Green function for the $\varphi_{\pi}$ field we obtain

$$
m_{\pi}^{2}(T, \mu) \equiv-i c^{2}(T, \mu)\left[D_{\pi}(0)\right]_{\beta}^{-1}=\left.c^{2}(T, \mu) \frac{\partial^{2} V}{\partial \pi^{2}}\right|_{m i n}=\left.\frac{c^{2}(T, \mu)}{c_{o}^{2}} \frac{\partial^{2} V}{\partial \varphi_{\pi}^{2}}\right|_{m i n}
$$

where $c^{2}(T, \mu)$ is the residue of $D_{\pi}\left(p^{2}\right)$ at the pole. Using the explicit form of the effective potential one has

$$
m_{\pi}^{2}(T, \mu)=-c^{2}(T, \mu) \frac{M}{g \hat{\sigma}}
$$

Therefore, from eq. (4.11) we have

$$
\begin{gathered}
f_{\pi}(T, \mu)=-2 \frac{c_{o}}{c^{2}(T, \mu)} \hat{\sigma} \\
m_{\pi}^{2}(T, \mu) f_{\pi}^{2}(T, \mu)=-4 \frac{M}{g^{2}} \frac{c_{o}^{2}}{c^{2}(T, \mu)} g \hat{\sigma}
\end{gathered}
$$

Eq.(4.17) is just the Adler-Dashen relation at finite temperature and chemical potential. By comparing it to the $T=\mu=0$ case

$$
m_{\pi}^{2} f_{\pi}^{2}=-4 \frac{M}{g^{2}} g \hat{\sigma}
$$

\footnotetext{
${ }^{3}$ This is the case in which the $\pi$ propagator can be approximated as $i c_{o}^{2} /\left(p^{2}-m_{\pi}^{2}\right)$.
} 
we see that (4.18) is modified by the term $c_{0}^{2} / c^{2}(T, \mu)$ which depends explicitly on the temperature and chemical potential (beside the implicit dependence contained in $g \hat{\sigma}$ ).

At this point we can obtain $m_{\pi}(T, \mu)$ and $f_{\pi}(T, \mu)$ by calculating explicitly the factor $c(T, \mu)$. If we remember that the inverse propagator is defined in general as

$$
D^{-1}\left(p^{2}\right)=i(1+\delta Z)-\Pi\left(p^{2}\right)
$$

we can find $c(T, \mu)$ by evaluating the self-energy for the $\pi$ field as given by the diagram shown in Fig. 14. Carrying on the calculation as done in App.C we finally arrive at

$$
\frac{1}{c(T, \mu)^{2}}=\frac{N}{4 \pi \hat{\sigma}^{2}}\left[1+2 \hat{\sigma}^{2} \frac{d}{d \hat{\sigma}^{2}} \int \frac{d q}{2 \omega_{q}}\left(\frac{1}{e^{\beta\left(\omega_{q}+\mu\right)}+1}+\frac{1}{e^{\beta\left(\omega_{q}-\mu\right)}+1}\right)\right]
$$

where we have defined $\omega_{q}=\sqrt{q^{2}+g^{2} \hat{\sigma}^{2}}$.

We notice that eq. (4.20) can also be verified, at least in the chiral limit, by evaluating explicitly the axial current matrix element. Indeed, for $m_{\pi}=0$, we obtain

$$
i p_{\mu} f_{\pi}=\left\langle 0\left|\mathcal{J}_{\mu}^{5}\right| \varphi_{\pi}\right\rangle=-2 \frac{c_{0}}{c(T, \mu)^{2}} \hat{\sigma} p_{\mu}
$$

The behaviours of $f_{\pi}$ and $m_{\pi}$ in $T$ and $\mu$ are shown respectively in Figs. 15, 16 and Figs. 17, 18. Notice that, if $\alpha=0$ could be taken as physically meaningful, $f_{\pi}$ could be used as a physical signal (it is a measurable quantity) of spontaneous symmetry breaking (for $T<T_{c}$ ) and of its restoration (for $T>T_{c}$ ). Unfortunately this is not the case, because massless bosons in two dimensions are not allowed. Anyway we notice that for small values of $\alpha$ we can still distinguish the transition region where $f_{\pi}$ decreases very rapidly.

As far as the pion mass is concerned we can say that $m_{\pi}$ grows up quickly for $T>T_{c}$, showing that in this case one is outside the range of validity of the soft pion hypothesis and the behaviour shown in Fig. 16 keeps only a qualitative value. Nevertheless we expect the pion mass to be independent of the symmetry breaking parameter (supposed be small) once the symmetry is restored, because in such a case the main parameter would be the scale of the theory. On the other hand for $T<T_{c}$, in the case $\alpha=0$, the symmetry would be spontaneously broken and the pion would be the associated Goldstone boson; the mass that it acquires is thus strongly dependent on the symmetry breaking parameter $\alpha$. Anyway a more precise analysis, that we are not interested to carry on in this paper, could be carried out by considering the full dependence of the pion propagator on $p^{2}$. 


\section{Discussion and Conclusions}

Study of QCD at finite temperature and density is a formidable problem both for analytical methods and for numerical simulations. It may then be of interest to perform similar studies on simpler models to get an introductory experience in view of the harder QCD problems and to have some hints at the type of phenomena that may be present. The most easily treatable problems are in dimensions lower than 4, particularly in 2 dimensions, one of space one of time. Unfortunately the lowest dimensionality brings out peculiar features in itself, which have been known since some time, and are particularly expressed by the contents of the so-called Mermin-Wagner Coleman theorem.

A number of analogies, among them asymptotic freedom, suggest the Gross-Neveu model in two dimensions as an interesting candidate in order to carry out orientation studies of what might happen to the physical QCD problem at finite temperatures and densities. In its original massless form the model falls into the theoretical problems caused by its low dimensionality, with direct consequences on symmetry breaking and phase structure, on the validity of an expansion in the inverse of the number of flavours $(1 / N$ expansion), and on the reliability of a mean field approximation.

The simplest massless Gross-Neveu model has a discrete $\gamma_{5}$ symmetry, spontaneously broken at zero temperature and zero chemical potential, without contradiction of the no-go theorems for one-space and one-time dimensions. One expects however symmetry restoration as soon as $T \neq 0$, for any finite value of $N$, through a kink-antikink formation mechanism. One can consider using a mean field approximation only for the strict $N \rightarrow \infty$ limit, where kinks become suppressed and a nominal phase transition takes place at finite $T$.

When the chemical potential $\mu$ is also non vanishing, the phase diagram for $N \rightarrow \infty$ shows a structure involving a tricritical point. Our main interest is the massive case, for which chiral symmetry is never valid and strictly the no-go theorems do not apply. The expected suppression of kink-antikink configurations will restore the validity of the large $N$ limit, and one finds that some first order phase transition survives in the $T-\mu$ plane in spite of the explicit breaking of chiral symmetry, with a critical line apparently persisting also for large masses.

The phase diagram for the massive Gross-Neveu model has been shown in Fig. 3, for different values of the mass parameter. Beyond some (mass-dependent) value of the chemical potential $\mu$ the fermion condensate shows a discontinuity at a critical value of $T$, which becomes smaller for higher $\mu$. One has thus lines of first order transitions.

There are no second order transition lines for finite mass values, but the condensate variation appears as very steep for small masses. 
Corresponding to the first order transitions one finds a latent heat visible in the diagram for the energy density. The phase diagram can, perhaps more physically, be regarded on the plane (inverse fermion density)-pressure, or alternatively on the plane (fermion density)-(energy density). The isotherms in the former plane have the aspect of the popular Van der Waals curves of water, showing vapour-liquid transition. These Van der Waals curves are here directly provided by the formalism, without having to apply Maxwell construction. The end point of the region of phase coexistence is a critical point. The massive model admits a Landau expansion, as there are no problems arising from possible long range fluctuations. From the equality of the pressure at the borders one obtains Clausius-Clapeyron like relations.

A massless Gross-Neveu model exists also with continuous chiral symmetry. For vanishing $T$ and $\mu$ uncritical use of the mean field approximation would lead analytical results translating those of the corresponding discrete symmetry model. One knows however that there cannot be Goldstones in two dimensions (Coleman theorem). This is reflected by the appearance of infrared divergent pion correlations at the immediately non leading order in the $1 / N$ expansion. An almost long range order, corresponding to power law correlation decrease, and reminiscent of the Kosterlitz-Thouless behaviour of the $X-Y$ spin model, is however expected, also on simple dimensional arguments. The " almost long range order " does not survives at any finite $T$, even for large $N$. Only if $N \rightarrow \infty$ is intended as constructive definition of a distinct new model one obtains the mean field description. Such constructed model, where fluctuations have automatically been suppressed, may not be a consistent approximation to a massless Lagrangian model.

The $N \rightarrow \infty$ properties can be analyzed by a comparison of the partition functions after and before symmetry rotation, illustrating the kind of dimensional reduction occurring in a thermal system, and evidencing at the same time the role played by the infinite $N$. One can also discuss the large $N$ limit of the fluctuations in the infrared, through study of the massless thermal propagator.

From the point of view of constructing some treatable approximation to physical QCD, suppressing fluctuations may bring closer to the real situation. Independently of this consideration our interest has been centered on massive models, which strictly escape the no-go theorems of two dimensions. QCD itself has massive quarks, and the general warning when passing to some massless limit is that this is certainly more dangerous in any comparable two dimensional model than in QCD itself. Nevertheless we get from our exercise the conviction that an overall physical content is included in the $N \rightarrow \infty$ limit. We had previously studied the chiral phase transition in QCD at finite temperature and density in a mean field composite model. The analogy of those QCD results to those provided by the $N \rightarrow \infty$ study of the massive Gross-Neveu model is quite remarkable and tends to support the impression of a possibly realistic phase space structure.

The model with continuous chiral symmetry, broken by the mass term, can easily 
be led back to the analogous discrete symmetry model to qualitatively discuss its phase diagram and its thermodynamics at finite $T$ and $\mu$. A main point of the continuous symmetry study has been to discuss the properties of the would-be Goldstone, which however here can never actualize itself as a Goldstone because of the low dimensionality. Nevertheless one can again build up for finite mass a kind of Adler-Dashen relation at finite $T$ and $\mu$, and derive the behaviours of the " pion decay constant " and of " pion mass ". One cannot, of course, use the pion-constant in this case to retrace the symmetry breaking, however we find that traces of the transition remains still visible in the finite mass model. In conclusion we can only insist on the overall impression of the usefulness of the Gross-Neveu model, in spite of its low dimensionality, to illustrate possible behaviours relevant to chiral phases of QCD at finite $T$ and $\mu$. We have carried out here a detailed study of such behaviours.

\section{Appendix}

\section{A. Expansion of the effective potential for small $\alpha$}

We show some useful formulas to derive the results of ref.[9] and to approximate our results when the mass parameter $\alpha$ is small. We remember that, whilst the effective potential we evaluate is a function which can be safely approximated for small $\alpha$, to set $\alpha=0$ is a different matter. In fact for the latter case one should take into account that the kinetic term of the effective action produces an ill-defined propagator. Thus, every result where $\alpha$ is "physically" taken as vanishing, serves only to recover some known result or to possibly suggest what would be expected in a $3+1$ dimensional model.

At finite $r$ and $\eta$ the effective potential is given by

$$
\begin{aligned}
\mathcal{V}(\omega) & =\frac{\pi}{2 \lambda} \omega^{2}(1+\delta Z)-r \sum_{n} \int_{0}^{+\infty} d k \ln \left[((2 n+1) \pi r-i \eta)^{2}+\omega^{2}+k^{2}\right]+\alpha \omega \\
& \equiv \mathcal{V}^{(0)}+\alpha \omega
\end{aligned}
$$

We notice that at any finite $r$ (and/or $\eta$ ), this expression is analytic in the limit $\omega \rightarrow 0$ (this is not true at $r=\eta=0$, due to a logarithmic divergence in the $n=0$ term). Thus we can perform a Landau expansion to reproduce the right structure of the effective potential near to the origin. We write

$$
\mathcal{V}\left(\omega^{2}\right) \simeq \frac{1}{2} a \omega^{2}+\frac{1}{4} b \omega^{4}+\frac{1}{6} c \omega^{6}+\alpha \omega \quad(\omega \simeq 0)
$$

where the expansion coefficients are given by

$$
\left.a \equiv 2 \frac{d \mathcal{V}^{(0)}}{d \omega^{2}}\right|_{\omega=0} ;\left.\quad b \equiv 2 \frac{d^{2} \mathcal{V}^{(0)}}{d\left(\omega^{2}\right)^{2}}\right|_{\omega=0} ;\left.\quad c \equiv \frac{d^{3} \mathcal{V}^{(0)}}{d\left(\omega^{2}\right)^{3}}\right|_{\omega=0}
$$


By carrying out explicitly the renormalization, from (3.14), we obtain

$$
a=1+\int_{0}^{+\infty} d k\left[\frac{k^{2}}{\left[k^{2}+1\right]^{\frac{3}{2}}}+\frac{1}{k}\left(\frac{1}{\mathrm{e}^{(k+\eta) / r}+1}+\frac{1}{\mathrm{e}^{(k-\eta) / r}+1}-1\right)\right]
$$

This expression can be integrated, as shown in App.B. We have

$$
a=\ln \pi r+\operatorname{Re}\left[2 \psi\left(\frac{i \eta}{r \pi}\right)-\psi\left(\frac{i \eta}{2 r \pi}\right)\right]
$$

Following this procedure also for $b$ and $c$ would lead to very complicated expressions. Anyway, by noticing that ultraviolet divergencies are present only in the $\omega^{2}$-term, we can evaluate the coefficients $b$ and $c$ directly from eq.(A.1). Carrying out the integration in $k$, after having derived once, and then deriving again, we find

$$
\begin{gathered}
b=\frac{1}{8} \frac{1}{\pi^{2} r^{2}} \operatorname{Re} \sum_{n=0}^{+\infty} \frac{1}{[n+1 / 2-i \eta / 2 \pi r]^{3}}=\frac{1}{8} \frac{1}{\pi^{2} r^{2}} \operatorname{Re} \xi\left(3, \frac{1}{2}-\frac{i \eta}{2 \pi r}\right) \\
c=-\frac{3}{4}\left(\frac{1}{2 \pi r}\right)^{4} \operatorname{Re} \sum_{n=0}^{+\infty} \frac{1}{[n+1 / 2-i \eta / 2 \pi r]^{5}}=-\frac{3}{4}\left(\frac{1}{2 \pi r}\right)^{4} \operatorname{Re} \xi\left(5, \frac{1}{2}-\frac{i \eta}{2 \pi r}\right)
\end{gathered}
$$

Using now the relations (A.5) and (A.6), and referring to Fig. 2 , we can easily obtain the following results

- The equation for the line $A P_{t} D$ (see Treml in ref. [11]), solution of $a=0$

$$
r=\frac{1}{\pi} \exp \left\{-\operatorname{Re}\left[2 \psi\left(\frac{i \eta}{r \pi}\right)-\psi\left(\frac{i \eta}{2 r \pi}\right)\right]\right\}
$$

- The coordinates of the tricritical point $P_{t}$, determined by the solutions of the equations $a=0, b=0$ 用

$$
r_{t}=0.318, \quad \eta_{t}=0.608
$$

- For $\eta=0$ the effective potential becomes $(\epsilon \equiv \omega / r)$

$$
\mathcal{V}(\omega, r, \eta=0)=\frac{1}{4} \omega^{2}\left(\ln \omega^{2}-1\right)+\alpha \omega-2 r^{2} \int_{0}^{+\infty} d k \ln \left(1+\mathrm{e}^{-\sqrt{k^{2}+\epsilon^{2}}}\right)
$$

Expanding it in $\epsilon^{2}$ (see ref. 16]) we arrive at

$$
\mathcal{V}(\omega) \simeq \frac{1}{2}(\ln \pi r-\gamma) \omega^{2}+\frac{1}{4} \frac{7}{8 \pi^{2}} \frac{\xi(3)}{r^{2}} \omega^{4}+\alpha \omega+\ldots
$$

where $\gamma=0.577 \ldots$ is the Euler constant. In this case it is sufficient to terminate the expansion at the order $\omega^{4}$, because the fact that $b$ is positive assures that the potential is bounded from below. For $\alpha=0$ the equation $a=0$ gives us the critical temperature 5

$$
T_{c}=\frac{m_{0}}{\pi} \mathrm{e}^{\gamma} \simeq 0.567 m_{0}
$$

\footnotetext{
${ }^{4}$ By inserting such results in the expression for $c$ we can verify that $c\left(r_{t}, s_{t}\right)>0$, assuring in this way the validity of the previous result.
} 
It is evident that the effective potential (A.1), since it admits the expansion (A.2) with IR finite coefficients, leads to the standard mean field critical exponents for the description of the system in the vicinity of the line $L_{I I}$ (up to the tricritical point included).

We notice that the equations that determine the tricritical point can be obtained by another method, based on the analysis of the phase diagram structure, specifically on the fact that the line $P_{t} E$ is the envelope of the solutions outside the origin of the stationarity equation for the potential. In other words (see Fig. 2), if we consider such solutions, it turns out that in the region $A P_{t} D O$ there is one and only one curve for each point (there is just one minimum ${ }^{5}$ ), while in $P_{t} D E$ there are two solutions for each point (a maximum and a minimum). Therefore the place where such points became coincident defines a curve, tangent to the former, that is just the line $P_{t} E$.

Such a curve can be parametrized by the value $\omega$ that makes the potential stationary in the interval $[0,1]$ (by the way we notice that $\omega$ is a continuous function of $T$ and $\mu$ ). In particular if $F(r, s, \omega)$ is the equation for the stationary points (outside the origin), the envelope curve is given by

$$
\left\{\begin{array}{l}
F(r, s, \omega)=0 \\
\frac{\partial F(r, s, \omega)}{\partial \omega}=0
\end{array}\right.
$$

and the tricritical point can be found by imposing $\omega=0$

These equations (evaluated in $\omega=0$ ) are formally equal to the equations $a=0, b=0$. In fact if we notice that

$$
\frac{\partial \mathcal{V}}{\partial \omega}=\omega F=2 \omega \frac{\partial \mathcal{V}}{\partial \omega^{2}} \quad \frac{\partial F}{\partial \omega}=4 \omega \frac{\partial^{2} \mathcal{V}}{\partial\left(\omega^{2}\right)^{2}}
$$

it comes out that the equation $\partial \mathcal{V} / \partial \omega^{2}=0$ determines the stationary points outside the origin, while $\omega\left[\partial^{2} \mathcal{V} / \partial\left(\omega^{2}\right)^{2}\right]=0$ is just the derivative of the former. Moreover, the second equation must be valid for every value of the parameter $\omega$ in the range $[0,1]$, and then it reduces to $\partial^{2} \mathcal{V} / \partial\left(\omega^{2}\right)^{2}=0$.

These two equations tell us that the envelope curve is the place where the curvature in the minimum outside the origin changes (the effective potential becomes like a parabola, and such minimum disappears), and in the $\omega=0$ limit they reduce to $a=0, b=0$.

\section{B. Coefficient $a$ of the Landau expansion}

In this section we show how to carry out the integrations in eq.(A.4) and thus obtain eq.(A.5). The first equation can be transformed according to the following formula

$$
\int_{0}^{+\infty} d y f(y)\left(\frac{1}{\mathrm{e}^{y-\lambda}+1}+\frac{1}{\mathrm{e}^{y+\lambda}+1}\right)=
$$

\footnotetext{
${ }^{5}$ Due to the symmetry we are just considering what happens, for examples, for $\omega \leq 0$.
} 


$$
\begin{aligned}
& =\int_{0}^{+\infty} d x(f(x+\lambda)+f(x-\lambda)) \frac{1}{\mathrm{e}^{x}+1}-\int_{0}^{\lambda} d x(f(x-\lambda)+f(\lambda-x)) \frac{1}{\mathrm{e}^{x}+1} \\
& +\int_{0}^{\lambda} d x f(x)
\end{aligned}
$$

from which we obtain

$$
a=1+\int_{0}^{+\infty} d y \frac{y^{2}}{\left[y^{2}+\alpha\right]^{3 / 2}}-\int_{\lambda}^{+\infty} d y \frac{1}{y}+P \int_{0}^{+\infty} d x \frac{2 x}{x^{2}-\lambda^{2}} \frac{1}{\mathrm{e}^{x}+1}
$$

The first two integrations give us

$$
a=\ln \left(\frac{2 \lambda}{\alpha}\right)+\int_{0}^{+\infty} d x \frac{2 x}{x^{2}-\lambda^{2}} \frac{1}{\mathrm{e}^{x}+1}
$$

The remaining integral can be transformed according to

$$
\begin{aligned}
P \int_{0}^{+\infty} d x \frac{2 x}{x^{2}-\lambda^{2}} \frac{1}{\mathrm{e}^{x}+1}= & P \int_{0}^{+\infty} d x \frac{2 x}{x^{2}-\lambda^{2}}\left(\frac{1}{\mathrm{e}^{x}-1}-\frac{2}{\mathrm{e}^{2 x}-1}\right) \\
& \equiv 2 I(\lambda)-4 I(2 \lambda)
\end{aligned}
$$

and

$$
\begin{aligned}
I(\lambda) & =P \int_{0}^{+\infty} d x \frac{x}{\mathrm{e}^{x}-1} \frac{1}{(x+\lambda)(x-\lambda)} \\
& =\lim _{\epsilon \rightarrow 0} \frac{1}{2} \int_{0}^{+\infty} d x \frac{x}{\mathrm{e}^{x}-1}\left(\frac{1}{(x+\lambda)(x-\lambda+i \epsilon)}+\frac{1}{(x+\lambda)(x-\lambda-i \epsilon)}\right)
\end{aligned}
$$

Using now the result 20]

$$
\int_{0}^{+\infty} d x \frac{x}{\left(x^{2}+\beta^{2}\right)\left(\mathrm{e}^{x}-1\right)}=\frac{1}{2}\left[\ln \left(\frac{\beta}{2 \pi}\right)-\frac{\pi}{\beta}-\psi\left(\frac{\beta}{2 \pi}\right)\right] \quad(\operatorname{Re} \beta>0)
$$

and carrying out the limit, we obtain

$$
2 I(\lambda)-4 I(2 \lambda)=\ln \frac{\pi}{\lambda}+\operatorname{Re}\left[2 \psi\left(\frac{i \lambda}{\pi}\right)-\psi\left(\frac{i \lambda}{2 \pi}\right)\right]
$$

from which is easy to get the wanted result.

\section{Pion self-energy}

From Fig. 14 we have

$$
\begin{aligned}
\Pi\left(p^{2}\right) & =N(-g)^{2} \int_{k}(-1) \operatorname{tr}\left[\gamma^{5} \frac{i}{\hat{p}+\hat{k}-m} \gamma^{5} \frac{i}{\hat{k}-m}\right] \\
& =2 \lambda \int_{k} \frac{m^{2}-k(k+p)}{\left((p+k)^{2}-m^{2}\right)\left(k^{2}-m^{2}\right)}
\end{aligned}
$$

By using the usual Feynman parameterization we can write

$$
\Pi\left(p^{2}\right)=2 \lambda \int_{0}^{1} d \alpha \int_{q} \frac{m^{2}-q^{2}+p^{2} \alpha(1-\alpha)}{\left[q^{2}+p^{2} \alpha(1-\alpha)-m^{2}\right]^{2}}
$$


In the soft pion limit we can write

$$
\Pi\left(p^{2}\right)=\Pi_{o}+p^{2} \Pi_{1}+\ldots
$$

where

$$
\Pi_{o}=-2 \lambda \int_{q} \frac{1}{q^{2}-m^{2}} \quad \Pi_{1}=\int_{q} \frac{1}{\left(q^{2}-m^{2}\right)^{2}}
$$

The inverse of the pion propagator residue on the pole is just $-i \Pi_{1}$. This can be evaluated using the Poisson summation

$$
\frac{1}{\beta} \sum_{n} f\left[(2 n+1) \frac{\pi}{\beta}+i \mu\right]=\sum_{n}(-1)^{n} \int \frac{d q_{o}}{2 \pi} f\left(q_{o}\right) \mathrm{e}^{i n \beta\left(q_{o}-i \mu\right)}
$$

We have

$$
\begin{aligned}
\Pi_{1} & =i \lambda \int \frac{d q}{2 \pi} \sum_{n}(-1)^{n} \int \frac{d q_{o}}{2 \pi} \frac{\mathrm{e}^{i n \beta\left(q_{o}-i \mu\right)}}{\left(q_{o}^{2}+q^{2}+m^{2}\right)^{2}} \\
& =\frac{i \lambda}{4 \pi m^{2}}+2 i \lambda \int \frac{d q}{2 \pi} \sum_{n=1}^{+\infty}(-1)^{n}(-1) \frac{d}{d m^{2}} \int \frac{d q_{o}}{2 \pi} \frac{\mathrm{e}^{i n \beta\left(q_{o}-i \mu\right)}}{\left(q_{o}^{2}+q^{2}+m^{2}\right)}
\end{aligned}
$$

The integral in $d q_{0}$ can be performed using the Cauchy theorem, and the sum turns out to be just a geometric series. At the end we arrive at

$$
\Pi_{1}=\frac{i}{c^{2}(T, \mu)}=\frac{i N}{4 \pi \hat{\sigma}^{2}}+2 i N \frac{d}{d \sigma^{2}} \int \frac{d q}{2 \pi} \frac{1}{2 \omega_{q}}\left(\frac{1}{e^{\beta\left(\omega_{q}+\mu\right)}+1}+\frac{1}{e^{\beta\left(\omega_{q}-\mu\right)}+1}\right)
$$

\section{Bosonic free propagators}

\section{Zero temperature}

We recall some known results at zero temperature.

In $D$ euclidean dimensions, the free two-point function in coordinates space

$$
D_{0}\left(x, m^{2}\right)=\int \frac{d^{D} k}{(2 \pi)^{D}} \frac{e^{-i k \cdot x}}{k^{2}+m^{2}}
$$

satisfies the wave equation

$$
\left(\square_{D}-m^{2}\right) D_{0}\left(x, m^{2}\right)=-\int \frac{d^{D} k}{(2 \pi)^{D}} e^{-i k \cdot x}=-\delta^{D}(x)
$$

where

$$
\square_{D} f=\sum_{\mu=1}^{D} \frac{\partial}{\partial x^{\mu}} \frac{\partial}{\partial x^{\mu}} f
$$


Lorentz invariance requires that $D_{0}\left(x, m^{2}\right)$ depends only on $r=\sqrt{x_{\mu} x_{\mu}}$; therefore (D.2) becomes

$$
\left[\frac{d^{2}}{d r^{2}}+\frac{D-1}{r} \frac{d}{d r}-m^{2}\right] D_{0}\left(r, m^{2}\right)=-\delta^{D}(x)
$$

By using the identity

$$
\frac{1}{k^{2}+m^{2}}=\int_{0}^{\infty} d \alpha e^{-\alpha\left(k^{2}+m^{2}\right)}
$$

in eq.(D.1), one gets

$$
D_{0}(r, m)=\frac{1}{(2 \pi)^{D / 2}} \frac{K_{\frac{D}{2}-1}(m r)}{\left[r^{(D-2) / 2} m^{(2-D) / 2}\right]}
$$

where use has been made of the representation for the modified Bessel functions

$$
\begin{aligned}
K_{\nu}(z)= & \frac{1}{2}\left(\frac{z}{2}\right)^{\nu} \int_{0}^{\infty} t^{-\nu-1} e^{-t-z^{2} / 4 t} d t \\
& |\arg z|<\pi / 2 ; \quad \operatorname{Re} z^{2}>0
\end{aligned}
$$

which behave [19], for fixed $\nu$ and $z \rightarrow 0$

$$
\begin{aligned}
& K_{0}(z) \sim-\ln \left(\frac{z}{2}\right)-\gamma \\
& K_{\nu}(z) \sim \frac{1}{2} \Gamma(\nu)\left(\frac{z}{2}\right)^{-\nu} \quad ; \quad(\operatorname{Re} \nu>0)
\end{aligned}
$$

where $\gamma=0.577 \ldots$ is the Euler's constant.

These formulas allow to recover the well known behaviours of the free massless propagators in $\mathrm{D}=2+1$ and $\mathrm{D}=3+1$ at zero temperature

$$
\begin{aligned}
D_{0}(r) & =\frac{1}{4 \pi} \frac{1}{r} \\
D_{0}(r) & =\frac{1}{4 \pi^{2}} \frac{1}{r^{2}}
\end{aligned}
$$

For $D=1+1$, performing the $m \rightarrow 0$ limit with fixed $r$, from (D.8) one finds that the expression for the free massless propagator in two (infinite) dimensions

$$
D_{0}(r)=-\frac{1}{2 \pi} \ln (r)-\frac{1}{2 \pi} \ln \left(\frac{m}{2}\right)-\frac{\gamma}{2 \pi}
$$

is logarithmically divergent for large distances.

\section{Finite temperature free propagators in $\mathrm{D}=\mathrm{d}+1$ dimensions}

By working in the imaginary times formalism, the passage from Minkowski metrics to Euclidean metrics comes out naturally. In fact one has

$$
D_{\beta}(x)=\int_{k} \frac{i}{k^{2}-m^{2}+i \varepsilon} e^{-i k \cdot x}
$$


with

$$
\begin{aligned}
& k^{\mu}=\left(k_{0}, \mathbf{k}\right)=(2 \pi i n T, \mathbf{k}) \\
& x^{\mu}=\left(x_{0}, \mathbf{x}\right)=(-i \tau, \mathbf{x})
\end{aligned}
$$

$-\beta<\tau<\beta$, and

$$
\int_{k}=\frac{i}{\beta} \sum_{n=-\infty}^{+\infty} \int_{-\infty}^{+\infty} \frac{d^{d} k}{(2 \pi)^{d}}
$$

thus $(\beta=1 / T)$

$$
D_{\beta}(\tau, \mathbf{x})=T \sum_{n=-\infty}^{+\infty} \int_{-\infty}^{+\infty} \frac{d^{d} k}{(2 \pi)^{d}} \frac{e^{-i 2 \pi n T \tau+i \mathbf{k} \cdot \mathbf{x}}}{4 \pi^{2} n^{2} T^{2}+\mathbf{k}^{2}+m^{2}}
$$

One wants to evaluate the $\mathbf{x}$ behaviour in the massless limit for $D=1+1, D=2+1$ and $D=3+1$, and in particular the large distances behaviour in the massless limit. To this end it is sufficient to separate out the $n=0$ term in eq.(D.13) after having set $m=0$

$$
D_{\beta}(\tau, \mathbf{x})=T \int \frac{d^{d} k}{(2 \pi)^{d}} \frac{e^{i \mathbf{k} \cdot \mathbf{x}}}{\mathbf{k}^{2}}+\frac{1}{2 \pi^{2} T} \int \frac{d^{d} k}{(2 \pi)^{d}} e^{i \mathbf{k} \cdot \mathbf{x}} \sum_{n=1}^{+\infty} \frac{\cos (n y)}{n^{2}+\alpha^{2}}
$$

where $\alpha \equiv k /(2 \pi T)$ and $y \equiv 2 \pi T \tau$.

The sum of the series is

$$
\sum_{n=1}^{+\infty} \frac{\cos (n y)}{n^{2}+\alpha^{2}}=\frac{\pi}{2 \alpha} \frac{\operatorname{ch}[\alpha(\pi-y)]}{\operatorname{sh}(\alpha \pi)}-\frac{1}{2 \alpha^{2}}
$$

The large distances behaviours are determined by the IR leading term of (D.14). It is easy to see, by expanding (D.15) for small $k$, that the contribution of the sum (D.15) is subleading with respect to the first term on the r.h.s. in (D.14). Thus the free massless propagator in $\mathrm{D}=\mathrm{d}+1$ dimensions at finite temperature for large distances behaves simply as $T \times$ (free massless propagator in $\mathrm{d}$ dimensions at zero temperature).

Let us now evaluate the full $\mathbf{x}$ dependence for $d=1,2,3$.

$\mathrm{D}=1+1$

In this case

$$
D_{\beta}(\tau, x)=T \sum_{n=-\infty}^{+\infty} \int_{-\infty}^{+\infty} \frac{d k}{2 \pi} \frac{e^{-i 2 \pi n T \tau+i k x}}{4 \pi^{2} n^{2} T^{2}+k^{2}+m^{2}}
$$

and it is evident that the zero mass limit is badly divergent. Anyway this expression has a well defined behaviour in $x$ as it can be easily seen by the fact that its derivative with 
respect to $x$ is a convergent integral even for $m=0$.

By using the result (D.15) the massless propagator can be put in the form

$$
D_{\beta}(\tau, x)=\frac{1}{2 \pi} \int_{0}^{+\infty} \frac{d k}{k} \cos (k x) \frac{\operatorname{ch}\left[k\left(\frac{\beta}{2}-\tau\right)\right]}{\operatorname{sh}\left(k \frac{\beta}{2}\right)}
$$

As expected the IR behaviour of this integral is the same as that of the $n=0$ term of (D.16).

Let us now evaluate the $x$ derivative of (D.17)

$$
\begin{aligned}
\frac{\partial D_{\beta}(\tau, x)}{\partial x} & =-\int_{0}^{+\infty} \frac{d k}{2 \pi} \frac{\sin (k x) \operatorname{ch}\left[k\left(\frac{\beta}{2}-\tau\right)\right]}{\operatorname{sh}\left(k \frac{\beta}{2}\right)} \\
& =-\frac{T}{2} \frac{\operatorname{sh}(2 \pi x T)}{\operatorname{ch}(2 \pi x T)-\cos (2 \pi \tau T)}
\end{aligned}
$$

Integrating again one gets

$$
D_{\beta}(\tau, x)=C(T)-\frac{1}{4 \pi} \ln [\operatorname{ch}(2 \pi T x)-\cos (2 \pi T \tau)]
$$

where $C(T)$ is an integration constant which does not depend on $x$. It is easy to verify that in the $T \rightarrow 0$ limit it satisfies ( $\mathrm{D} .10)$ in the coordinate dependence in two dimensions at zero temperature, whereas at finite $T$, for $|x| \rightarrow \infty$ the leading behaviour is that expected for one dimension

$$
D_{\beta}(\tau, x) \sim-\frac{T|x|}{2}
$$

$\mathrm{D}=2+1$

We must evaluate

$$
\begin{aligned}
D_{\beta}(\tau, \mathbf{x}) & =T \sum_{n=-\infty}^{+\infty} \int_{-\infty}^{+\infty} \frac{d^{2} k}{(2 \pi)^{2}} \frac{e^{-i 2 \pi n T \tau+i \mathbf{k} \cdot \mathbf{x}}}{4 \pi^{2} n^{2} T^{2}+\mathbf{k}^{2}+m^{2}} \\
& =\frac{T}{2 \pi} \sum_{n=-\infty}^{+\infty} \int_{0}^{+\infty} \frac{k J_{0}(k x) e^{-i 2 \pi n T \tau}}{4 \pi^{2} n^{2} T^{2}+k^{2}+m^{2}} d k
\end{aligned}
$$

Separating out the $n=0$ term (from here on remember that $x \equiv|\mathbf{x}|$ ), one gets

$$
D_{\beta}(\tau, x)=\frac{T}{2 \pi} K_{0}(m x)+\frac{T}{\pi} \sum_{n=1}^{\infty} \cos (2 \pi n T \tau) K_{0}\left(x \sqrt{4 \pi^{2} n^{2} T^{2}+m^{2}}\right)
$$


The sum of the series for $m=0$ is not a closed form

$$
\begin{aligned}
\sum_{n=1}^{\infty} \cos (n y) K_{0}(n z)= & \frac{\pi}{2} \frac{1}{\sqrt{z^{2}+y^{2}}+\frac{1}{2}\left[\gamma-\ln \left(\frac{4 \pi}{z}\right)\right]} \\
& +\frac{\pi}{2} \sum_{n=1}^{\infty}\left[\frac{1}{\sqrt{z^{2}+(2 \pi n-y)^{2}}}-\frac{1}{2 \pi n}\right] \\
& +\frac{\pi}{2} \sum_{n=1}^{\infty}\left[\frac{1}{\sqrt{z^{2}+(2 \pi n+y)^{2}}}-\frac{1}{2 \pi n}\right] \quad ; \quad z>0, y \in R
\end{aligned}
$$

$(\gamma=0.577 .$. is again the Euler's constant)

Identifying $y \equiv 2 \pi T \tau, z \equiv 2 \pi T x$, and taking into account that, as already seen in (D.7), for $m \rightarrow 0$

$$
K_{0}(m z) \sim-\ln \left(\frac{m z}{2}\right)-\gamma
$$

it follows that for $m \rightarrow 0$ the propagator is given by the expression

$$
\begin{aligned}
D_{\beta}(\tau, x) \sim & -\frac{T}{2 \pi} \ln (m)+\frac{T}{2 \pi} \ln (T) \\
& +\frac{1}{4 \pi} \frac{1}{\sqrt{x^{2}+\tau^{2}}} \\
& +\frac{T}{4 \pi} \sum_{n=1}^{\infty}\left[\frac{1}{\sqrt{T^{2} x^{2}+(n-T \tau)^{2}}}-\frac{1}{n}\right] \\
& +\frac{T}{4 \pi} \sum_{n=1}^{\infty}\left[\frac{1}{\sqrt{T^{2} x^{2}+(n+T \tau)^{2}}}-\frac{1}{n}\right]
\end{aligned}
$$

In the second line we see the term which survives for $T \rightarrow 0$, which, as expected, corresponds to the first term of (D.8).

For $T \neq 0$, the dominant term for $x \rightarrow \infty$ comes from summation of the series. We can use the summation formula 22

$$
\begin{aligned}
\sum_{n=a}^{N} f(n)= & \int_{a}^{N} f(x) d x+\frac{1}{2} f(a)+\frac{1}{2} f(N) \\
& +\sum_{s=1}^{q-1} \frac{B_{2 s}}{(2 s) !}\left[f^{(2 s-1)}(N)-f^{(2 s-1)}(a)\right]+R_{q}(N)
\end{aligned}
$$

where $a, q, N$, are arbitrary integers with $a<N$ and $q>0$, and

$$
R_{q}(N)=\frac{B_{2 q}}{(2 q) !}\left[f^{(2 q-1)}(N)-f^{(2 q-1)}(a)\right]-\int_{a}^{N} \frac{B_{2 q}(x-[x])}{(2 q) !} f^{(2 q)}(x) d x
$$

This formula holds if $f^{(2 q)}(x)$ is absolutely integrable over $(a, N)$. The first Bernoulli coefficients are

$$
\frac{B_{2}}{2 !}=\frac{1}{12} ; \quad \frac{B_{4}}{4 !}=-\frac{1}{720}
$$


It is easy to see that for $x \rightarrow \infty$ the terms in the first line of (D.26) are dominant and that $\ln (m)$ is the only infinite constant for $m \rightarrow 0$. The final result, giving the large $x$ dependence in the massless limit, can be put in the form

$$
\begin{aligned}
D_{\beta}(\tau, x)= & -\frac{T}{2 \pi} \ln (m)+g(\tau, x, T) \\
& -\frac{T}{4 \pi} \ln \left[\beta+\tau+\sqrt{x^{2}+(\beta+\tau)^{2}}\right] \\
& -\frac{T}{4 \pi} \ln \left[\beta-\tau+\sqrt{x^{2}+(\beta-\tau)^{2}}\right]
\end{aligned}
$$

where

$$
\begin{aligned}
& g(\tau, x, T) \rightarrow 0 \text { per } x \rightarrow \infty \\
& g(\tau, x, T) \rightarrow \frac{1}{4 \pi} \frac{1}{\sqrt{x^{2}+\tau^{2}}} \text { for } T \rightarrow 0
\end{aligned}
$$

and thus, for $x \rightarrow \infty$

$$
D_{\beta}(\tau, x) \sim-\frac{T}{2 \pi} \ln (x)
$$

which is, as expected, the behaviour typical of two dimensions.

$\mathrm{D}=3+1$

In $\mathrm{D}=3+1$ the massless propagator is well defined even at finite temperature

$$
\begin{aligned}
D_{\beta}(\tau, \mathbf{x}) & =\frac{1}{\beta} \sum_{n=-\infty}^{+\infty} \int_{-\infty}^{+\infty} \frac{d^{3} k}{(2 \pi)^{3}} \frac{e^{-i 2 \pi n T \tau+i \mathbf{k} \cdot \mathbf{x}}}{4 \pi^{2} n^{2} T^{2}+\mathbf{k}^{2}} \\
& =\frac{T}{2 \pi^{2} x} \sum_{n=-\infty}^{+\infty} \int_{0}^{\infty} \frac{k \sin (k x)}{4 \pi^{2} T^{2} n^{2}+k^{2}} e^{-i 2 \pi n T \tau} d k
\end{aligned}
$$

By separating the $n=0$ term, and using again the sum of the series in (D.15), one easily arrives to the expression

$$
D_{\beta}(\tau, x)=\frac{T}{4 \pi x} \frac{\operatorname{sh}(2 \pi T x)}{\operatorname{ch}(2 \pi T x)-\cos (2 \pi T \tau)}
$$

For $T \rightarrow 0$ it is easy to recover the four dimensional result already given in the second of (D.8), whereas for $T \neq 0$ and $x \rightarrow \infty$ the leading behaviour is that of three dimensions

$$
D_{\beta}(\tau, x) \sim \frac{T}{4 \pi x}
$$




\section{References}

[1] D.J.Gross and A.Neveu, Phys. Rev. D10, 3235 (1974)

[2] N.D.Mermin and H.Wagner, Phys. Rev. Lett. 17,1133 (1966)

[3] S.Coleman, Comm. Math. Phys. 31, 259 (1973)

[4] A.Barducci, R.Casalbuoni, R.Gatto and G.Pettini, Phys. Rev. D49, 426 (1994)

[5] L.Jacobs, Phys. Rev. D10, 3956 (1974); B.Harrington and A.Yildiz, Phys. Rev. D11, 779 (1975)

[6] R.F.Dashen,S.K.Ma and R.Rajaraman, Phys. Rev. D11, 1499 (1975)

[7] E.Witten, Nucl. Phys. B145, 110 (1978)

[8] Y.Cohen, S.Elitzur and E.Rabinovici, Nucl. Phys. B220, 102 (1983)

[9] U.Wolff, Phys.Lett. 157B, 303 (1985)

[10] F.Karsch, J.Kogut and H.W.Wyld, Nucl. Phys. B280, 289 (1987)

[11] K.G.Klimenko, Z.Phys. C37, 457 (1988); T.F.Treml, Phys. Rev. D39, 679 (1989)

[12] B.Rosenstein, B.J.Warr and S.H.Park, Phys. Rep. 205, 59 (1991)

[13] V.L.Berezinski, JETP (Sov.Phys.) 32, 493 (1971); J.M.Kosterlitz and D.J.Thouless, J.Phys. C. 6, 1181 (1973)

[14] see also for instance A.D'Adda, P.Di Vecchia and M.Luscher, Nucl. Phys. B152, 125 (1979) A.C.Davis and A.M.Matheson, Nucl. Phys. B258, 373 (1985)

[15] see for instance J. Zinn-Justin, Quantum Field Theory and Critical Phenomena, Clarendon Press, Oxford (1989); G. Parisi, Statistical Field Theory, Addison-Wesley Publishing Company (1988)

[16] R.Jackiw, Phys.Rev. D9, 1686 (1974); L.Dolan and R.Jackiw, Phys.Rev. D9, 3320 (1974)

[17] See for instance:I.D.Lawrie and S.Sarbach, Phase Transitions and critical phenomena, Ed. C.Domb and J.L.Lebowitz, vol. 9, Academic Press, (1984), pp.1,155

[18] H.Leutwyler, Phys. Lett. B284, 106 (1992); A.Barducci, R.Casalbuoni, G.Pettini and R.Gatto Phys. Lett. B301, 95 (1993)

[19] M.Abramovitz and I.A.Stegun, Handbook of Mathematical Functions, Dover Pub.Inc. (1972) 
[20] I.S.Gradshteyn and I.M.Ryzhik, Tables of integrals, series and products, Academic Press (1985)

[21] C.Itzykson and J.B.Zuber, Quantum Field Theory, McGraw-Hill (1985)

[22] F.W.Olver Asymptotics and special functions Academic Press, 1974 


\section{FIGURE CAPTIONS}

Fig. 1: Effective potential at $T=\mu=0$ for various values of $\alpha$.

Fig. 2: $\quad$ Phase diagram for the massless Gross-Neveu model.

Fig. 3: Phase diagram for the massive Gross-Neveu model.

Fig. 4: Condensate behaviour in $r$ at $\eta=0$ for $\alpha=0, \alpha=0.01$ and $\alpha=0.1$.

Fig. 5: Condensate behaviour in $\eta$ at $r=0$ for $\alpha=0, \alpha=0.01$ and $\alpha=0.1$.

Fig. 6: $\quad$ Plot of $\varepsilon / r^{2}$ and $p / r^{2}$ vs $r$ for $\eta=0$ at $\alpha=0$.

Fig. 7: $\quad$ Plot of $\varepsilon / r^{2}$ and $p / r^{2}$ vs $r$ for $\eta=0.63>\eta_{t}$ at $\alpha=0$.

Fig. 8: Plot of $[\varepsilon+\alpha] / r^{2}$ and $[p+\alpha] / r^{2}$ vs $r$ for $\eta=0$ at $\alpha=0.01$. Remember that $\varepsilon(r=0, \eta=0) \simeq-\alpha$ for $\alpha \ll 1$.

Fig. 9: Plot of $[\varepsilon+\alpha] / r^{2}$ and $[p-\alpha] / r^{2}$ vs $r$ for $\eta=0.65>\eta_{t}(\alpha)$ at $\alpha=0.01$. Remember that $\varepsilon(r=0, \eta=0) \simeq-\alpha$ for $\alpha \ll 1$.

Fig. 10: Isotherms of the massless Gross-Neveu model in the $(1 / n, p)$ plane.

Fig. 11: Isotherms of the massive Gross-Neveu model in the $(1 / n, p)$ plane for $\alpha=0.01$.

Fig. 12: Phase diagram for the massless Gross-Neveu model in the $(n, \varepsilon)$ plane.

Fig. 13: Phase diagram for the massive Gross-Neveu model in the $(n, \varepsilon)$ plane for $\alpha=0.01$.

Fig. 14: Pion self-energy at one loop order.

Fig. 15: Pion decay constant vs. temperature for $\alpha=0, \alpha=0.01$ and $\alpha=0.1$.

Fig. 16: Pion mass vs. temperature for $\alpha=0.01$ and $\alpha=0.1$.

Fig. 17: Pion decay constant vs. chemical potential for $\alpha=0.01$.

Fig. 18: Pion mass vs. chemical potential for $\alpha=0.01$. 
This figure "fig1-1.png" is available in "png" format from: http://arxiv.org/ps/hep-th/9406117v1 
This figure "fig2-1.png" is available in "png" format from: http://arxiv.org/ps/hep-th/9406117v1 
This figure "fig3-1.png" is available in "png" format from: http://arxiv.org/ps/hep-th/9406117v1 
This figure "fig1-2.png" is available in "png" format from: http://arxiv.org/ps/hep-th/9406117v1 
This figure "fig2-2.png" is available in "png" format from: http://arxiv.org/ps/hep-th/9406117v1 
This figure "fig3-2.png" is available in "png" format from: http://arxiv.org/ps/hep-th/9406117v1 
This figure "fig1-3.png" is available in "png" format from: http://arxiv.org/ps/hep-th/9406117v1 
This figure "fig2-3.png" is available in "png" format from: http://arxiv.org/ps/hep-th/9406117v1 
This figure "fig3-3.png" is available in "png" format from: http://arxiv.org/ps/hep-th/9406117v1 